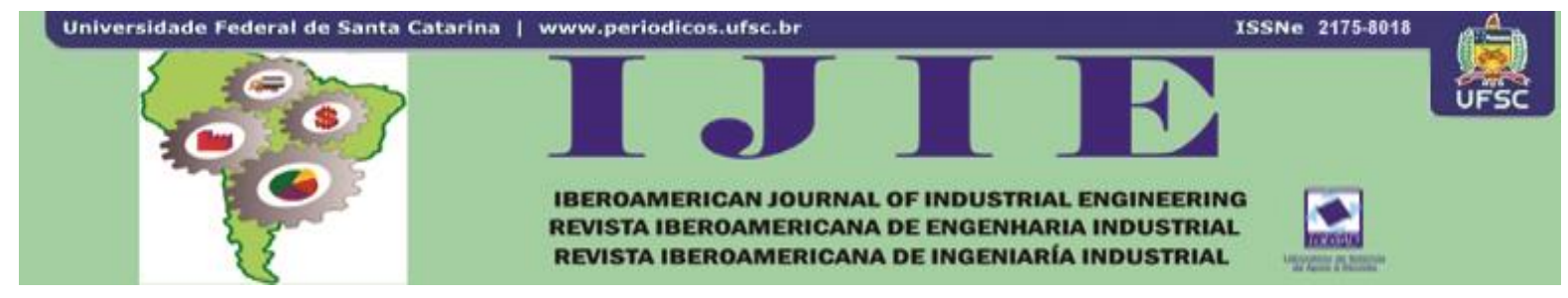

\title{
IMPROVING THE MATHEMATICAL FORMULATION FOR THE DETAILED SCHEDULING OF REFINED PRODUCTS PIPELINES BY ACCOUNTING FOR FLOW RATE DEPENDENT PUMPING COSTS
}

\author{
Vanina Guadalupe Cafaro ${ }^{1}$ \\ Diego Carlos Cafaro ${ }^{2}$ \\ Jaime Cerdá ${ }^{3}$
}

\begin{abstract}
This work presents a continuous-time mixed-integer linear programming (MILP) formulation to find the best detailed schedule for single-source multi-product pipelines, minimizing the total operating costs. By knowing the aggregate plan including pumping and delivery tasks, the best detailed solution tends to minimize pump stoppage/restart costs as well as pumping energy charges associated to the head loss inside the pipeline, which are strongly dependent on the flow rate at every pipeline segment. Pumping costs for transporting products into the pipeline are estimated by introducing a novel piecewise linear calculation of the energy loss. The proposed approach is applied to find the optimal detailed schedule for a real-world case study consisting of a single source pipeline with multiple offtake stations. Important reductions in the operational costs with regards to previous contributions are obtained.
\end{abstract}

Keywords: MILP approach. Detailed scheduling. Multiproduct pipeline. Friction head loss.

\section{INTRODUCTION}

Refined products pipelines usually connect refineries with distant distribution terminals located along the line. Each terminal comprises a collection of large tanks storing different refined products. Products move down the pipeline in batches. Sometimes the entire flow of the pipeline is diverted into a terminal tank, while in other occasions just a "split" or partial

\footnotetext{
1 Dra. en Ingeniería, Ingeniera Industrial, Instituto de Desarrollo Tecnológico para la Industria Química, Universidad Nacional del Litoral, Consejo Nacional de Investigaciones Científicas y Técnicas (INTEC, UNLCONICET), Güemes 3450, (3000) Santa Fe, Argentina. E-mail: vcafaro@fiq.unl.edu.ar.

${ }^{2}$ Dr. en Tecnología Química, Ingeniero Industrial Instituto de Desarrollo Tecnológico para la Industria Química, Universidad Nacional del Litoral, Consejo Nacional de Investigaciones Científicas y Técnicas (INTEC, UNL-CONICET), Güemes 3450, (3000) Santa Fe, Argentina. E-mail: dcafaro@fiq.unl.edu.ar.

3 Ph.D., Ingeniero Químico, Instituto de Desarrollo Tecnológico para la Industria Química, Universidad Nacional del Litoral, Consejo Nacional de Investigaciones Científicas y Técnicas (INTEC, UNL-CONICET), Güemes 3450, (3000) Santa Fe, Argentina. E-mail: jcerda@intec.unl.edu.ar.
}

Iberoamerican Journal of Industrial Engineering, Florianópolis, SC, Brasil, v. 5, n. 10, p. 115128, 2013. 
stream moves into the tank. From the terminal, petroleum products move to retail outlets or commercial and industrial consumers, commonly by tank cars.

The operation of a pipeline seems simple enough: pump fluid in one end and take it out on the other. While the principles dictating the behavior of fluids in pipelines are rather intuitive, the calculations involved can be fairly complex. Pressure makes fluids move. Pressure is a reflection of energy added to pipelines by pumps, compressors, or gravity.

The pressure in a shutdown (nonflowing) pipeline along a level route is the same along its entire length. For a shutdown line along the route with elevation changes, the pressure is higher in the valleys and lower at the hilltops. But once the batches start flowing into the pipeline, the pressure is almost always lower as the fluid moves along. Fluids always move from a point of higher energy (in pipelines energy is normally measured as pressure) to one of lower energy, unless something like a closed valve stops them. When energy is added to a pipeline by a pump or a compressor, pressure builds. If one opens any point, flow starts.

Major pipeline operating costs include: salaries; fuel and power; operating supplies; inspection and maintenance; insurance; local, state and federal taxes and fees. Power costs may be based on the local power provider's standard usage and rates. Since power costs are a significant percentage of overall operating costs, they may negotiate contracts with lower rates for off-peak usage or lower overall rates. They also might optimize the design to accommodate varying power plant rates (MIESNER; LEFFLER, 2006; LIANG; $\quad$ GONG; KANG, 2004).

The greatest feature of a multi-product pipeline is batch transportation. Pump connections at every power station, variations in the head loss caused by the movement of different products, and batch delivery/injection operations along the pipeline result in changes of the configuration of pump and valve sets of the whole pipeline. Different configurations of pump and valve sets impose diverse pipeline operation costs. Many studies have been performed on power cost optimization, especially concerning optimizing configurations of pump sets to achieve minimum pumping power cost while ensuring operation safety and satisfying the delivery requirements.

The optimal pump configuration in previous studies has been mainly determined by considering constraint conditions such as maximum and minimum suction and discharge pressures, pressures of high-elevation points, speed range of the control motor, and pump yield curves, with a constant electricity price assumption (LIANG; GONG; KANG, 2004) or 
even accounting for high electricity cost daily periods (REJOWSKI; PINTO, 2008) But none of them has been focused on the rigorous minimization of the head loss due to friction along the pipeline, which is strongly dependent on the flow rates.

\section{MILP FORMULATION FOR THE DETAILED SCHEDULING OF MULTIPRODUCT PIPELINES ACCOUNTING FOR THE HEAD LOSS}

In this section, we present the MILP optimization model providing the best detailed schedule of delivery operations, taking into account the energy pumping cost due to friction, by using a linear piecewise approximation to estimate the power required to move the fluid inside the pipeline.

\subsection{Nomenclature}

\subsubsection{Sets}

$I$ - Ordered set of batches ( $\left.I^{\text {old }} \cup I^{\text {new }}\right)$

$I^{\text {new }}$ - Set of new batches to be injected during the planning horizon

$I^{\text {old }}$ - Set of old batches in the pipeline at the beginning of the time horizon

$J$ - Set of terminals/pipeline segments

$J_{i, i^{\prime}}$ - Subset of depots demanding product from batch $i$ while injecting lot $i^{\prime}$

$K$ - Ordered set of detailed operations

$K_{i}-$ Subset of detailed operations taking place during the injection of batch $i$

$P-$ Set of products

$P_{i}-$ Product contained at each lot $i$

$R$ - Set of ranges in which the "power curve" is divided

$R_{j}$ - Flow ranges for each pipeline segment $j$

\subsubsection{Parameters}

$c a / c s$ - unit flow restart/ stoppage cost

$c p$ - unit pumping energy cost

$d d_{\mathrm{i}, \mathrm{j}}{ }^{\left({ }^{\prime}\right)}$ - aggregate delivery from batch $i$ to terminal $j$ during the injection of batch $i$ '

$f c o$ - fixed cost for performing a single pumping run

$f t_{i}, s t_{i}$, - completion/ starting time for the injection of batch $i$,

$p v$ - total pipeline volume

$q q_{i}$,- overall size of the new lot $i$,

Iberoamerican Journal of Industrial Engineering, Florianópolis, SC, Brasil, v. 5, n. 10, p. 115128, 2013. 
$\sigma_{j}-$ volumetric coordinate of depot $j$ from the origin of the pipeline

\subsubsection{Positive Variables}

$A V_{k}$ - Activated volume to perform operation $k$

$C D_{k}$ - Segment stoppage cost at operation $k$

$C_{k}$ - Completion time of the detailed operation $k$

$D_{i, j}{ }^{(k)}$ - Delivered volume of batch $i$ to terminal $j$ during run $k$

$F A T_{k}$ - Farthest active terminal receiving product during operation $k$

$F_{i, k}-\mathrm{F}$ inal coordinate of batch $i$ at the end of operation $k$

$L_{k}$ - Length of the detailed operation $k$

$L r_{j, r}{ }^{(k)}$ Equals $L_{k}$ if segment $j$ is active during operation $k$ and the volume is pumped at rate range $r$, and zero otherwise

$P C_{k} \quad$ Pumping cost due to friction loss during the detailed operation $k$

$Q j_{j, k} \quad$ Volume pumped through segment $j$ while performing the operation $k$

$Q_{k} \quad$ Injected volume during run $k$

$Q Q j_{j, r}{ }^{(k)}$ Equals $Q j_{j, k}$ if the volume is pumped at rate range $r$, and zero otherwise

$S V_{k}$ Stopped volume to perform operation $k$

$W_{i, k}$ Content of lot $i$ at the completion time of operation $k$

\subsubsection{Binary Variables}

$u_{k} \quad$ Denoting that operation $k$ is executed

$v_{j, k} \quad$ Equals 1 if the segment $(j-1, j)$ is active during run $k$

$x_{i, j}{ }^{(k)} \quad$ Indicating that a portion of batch $i$ is delivered to depot $j$ during operation $k$

$y_{j, r}{ }^{(k)}$ Denoting that the flow rate into segment $j$ during $k$ belongs to range $r$

\subsection{General Assumptions}

1. No elevation profile is considered. Although the inclusion of elevation data in the energy consumption equations would be relatively easy, they will be ignored for the sake of simplicity. We will assume a totally horizontal pipeline system.

2. The relationship between the energy loss due to friction and the pump rate $(q)$ will be approximated by a piecewise linear function. The head loss (in meters) into a pipeline of length $\ell$ and diameter $D$ can be derived from the Darcy Equation (A). 


$$
h_{L}[m]=f \frac{\ell}{D} \frac{v^{2}}{2 g}=f \frac{\ell}{D} \frac{q^{2}}{2 g}\left[\frac{4}{\pi D^{2}}\right]^{2}=8 f \frac{\ell}{D^{5}} \frac{q^{2}}{g \pi^{2}}
$$

Since refined products normally flow in turbulent regime into oil pipelines, the Fanning friction factor (f) should be calculated by the Colebrook-White equation (COLEBROOK; WHITE, 1937), which is an implicit function relating the friction factor with the pipeline roughness and the Reynolds number. For simplicity, we will assume constant physical properties (namely density and viscosity) of an average oil product transported through the system, so that the only variable in Equation (A) is the flow rate. Hence, the power required to compensate for the friction loss (in $\mathrm{kW}$ ) is given by Equation (B). It can be demonstrated that such equation represents a non-linear function, rapidly growing with q.

$$
P_{L}[k W]=8 f(q) \frac{\ell}{D^{5}} \frac{q^{2}}{g \pi^{2}} \frac{q \rho g}{10^{3}}=\frac{8}{10^{3}} f(q) \frac{\ell}{D^{5}} \frac{\rho q^{3}}{g \pi^{2}}
$$

In our model, we divide the flow rate interval $\left[q_{j}^{\min } ; q_{j}^{\max }\right]$ for every segment $j$ into $R$ ranges, and for each range we define a linear approximation of Equation (B).

$$
P_{L}[k W]=a_{j, r}+b_{j, r} q \quad \forall j \in J, r \in R
$$

Figure 1 shows a typical power curve depending on the flow rate, and the corresponding piecewise linear approximation defined for a particular oil product moved through a pipeline segment of the case study presented in the Section 3.

Figure 1 - Piecewise linear approximation of friction power consumption when moving a typical oil product through a pipeline segment

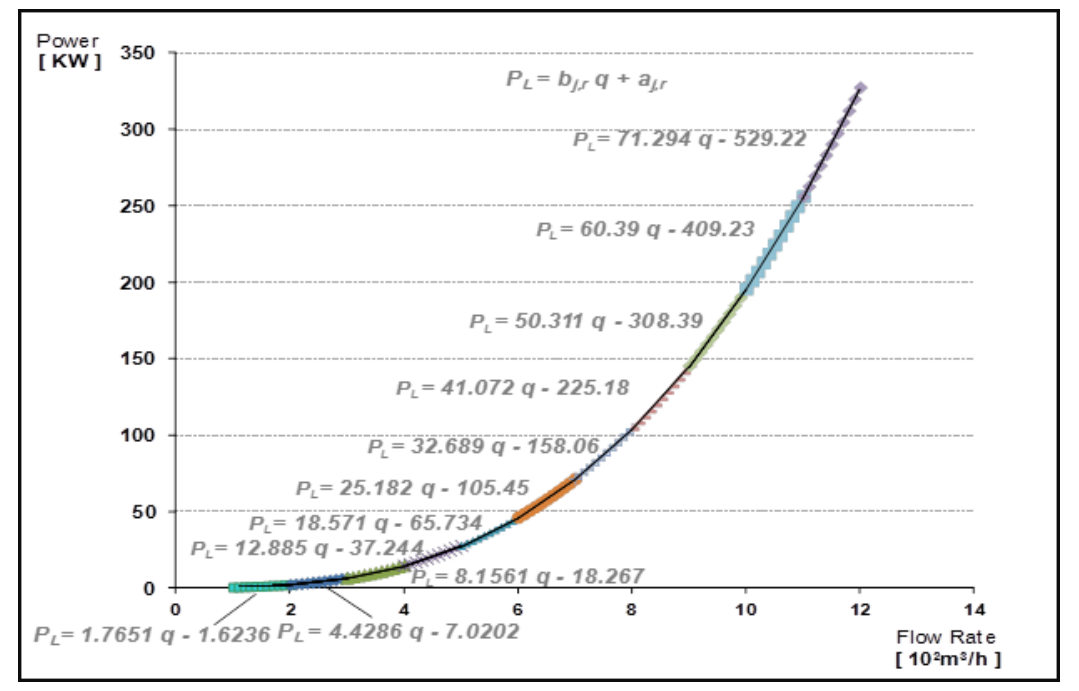

Iberoamerican Journal of Industrial Engineering, Florianópolis, SC, Brasil, v. 5, n. 10, p. 115128, 2013. 
Finally, the energy consumed at segment $j$, pumping $Q j_{j, k}$ volume units during the $L_{k}$ hours of operation $k$, at a flow rate belonging to range $r$, is estimated by Equation (D).

$$
E_{j, k}[k W h]=a_{j, r} L_{k}+b_{j, r} Q j_{j, k} \quad \forall j \in J, k \in K, r \in R \mid y_{j, r}{ }^{(k)}=1
$$

\subsection{Constraints}

- Start and ending times - Definition of the completion times and lengths of the detailed operations, chronologically arranged (Constraints 1-3).

$$
\begin{gathered}
C_{k} \leq f t_{i^{\prime}} \quad \forall i^{\prime} \in I^{\text {new }}, k=\operatorname{last}\left(K_{i^{\prime}}\right) \\
C_{k}-L_{k} \geq C_{k-1} \quad \forall k \in K \\
C_{k}-L_{k} \geq s t_{i^{\prime}} \quad \forall i^{\prime} \in I^{\text {new }}, k=\operatorname{first}\left(K_{i^{\prime}}\right)
\end{gathered}
$$

- Fictitious operations - Fictitious operations featuring null length at the optimum must be placed at the end of the run set to avoid solution degeneracy (Constraints 4-5).

$$
\begin{gathered}
u_{k} \leq u_{k-1} \quad \forall i^{\prime} \in I^{\text {new }}, k \in K_{i^{\prime}}, k>\text { first }\left(K_{i^{\prime}}\right) \\
l_{\text {min }} u_{k} \leq L_{k} \leq l_{\text {max }} u_{k} \quad \forall i^{\prime} \in I^{\text {new }}, k \in K_{i^{\prime}}
\end{gathered}
$$

- Tracking batch coordinates and sizes - The location and size of the batches inside the pipeline are monitored at the end of each operation (Constraints 6-8).

$$
\begin{gathered}
F_{i+1}{ }^{(k)}+W_{i}{ }^{(k)}=F_{i}{ }^{(k)} \quad \forall i \in I, i^{\prime} \in I^{\text {new }}, i^{\prime} \geq i+1, k \in K_{i^{\prime}} \\
W_{i}{ }^{(k)}=W_{i}^{(k-1)}-\sum_{j \in J_{i, i^{\prime}}} D_{i, j}{ }^{(k)} \quad \forall i \in I, i^{\prime} \in I^{\text {new }}, i^{\prime}>i, k \in K_{i^{\prime}} \\
W_{i^{\prime}}{ }^{(k)}=W_{i^{\prime}}{ }^{(k-1)}+Q_{k}-\sum_{j \in J_{i, i^{\prime}}} D_{i^{\prime}, j}{ }^{(k)} \quad \forall i^{\prime} \in I^{\text {new }}, k \in K_{i^{\prime}}
\end{gathered}
$$

- Imposing conditions for a product delivery - Product delivery restraints controlling the feasibility and the size of delivery operations are considered (Constraints 9-13).

$$
\begin{gathered}
d_{\min } x_{i, j}{ }^{(k)} \leq D_{i, j}{ }^{(k)} \leq d d_{i, j}{ }^{(k)} x_{i, j}{ }^{(k)} \\
\forall i \in I, i^{\prime} \in I^{\text {new }}, i^{\prime} \geq i, j \in J_{i, i^{\prime}}, k \in K_{i^{\prime}} \\
F_{i}^{(k-1)} \geq \sigma_{j} x_{i, j}{ }^{(k)} \quad \forall i \in I, i^{\prime} \in I^{\text {new }}, i^{\prime} \geq i, j \in J_{i, i^{\prime}}, k \in K_{i^{\prime}} \\
F_{i}^{(k)}-W_{i}^{(k)} \leq \sigma_{j}+\left(p v-\sigma_{j}\right)\left(1-x_{i, j}{ }^{(k)}\right) \\
\forall i \in I, i^{\prime} \in I^{\text {new }}, i^{\prime}>i, j \in J_{i, i^{\prime}}, k \in K_{i^{\prime}}
\end{gathered}
$$

Iberoamerican Journal of Industrial Engineering, Florianópolis, SC, Brasil, v. 5, n. 10, p. 115128, 2013. 


$$
\begin{aligned}
& \sum_{j \in J_{i, i^{\prime}}} D_{i, j}{ }^{(k)} \leq W_{i}{ }^{(k-1)} \quad \forall i \in I, i^{\prime} \in I^{\text {new }}, i^{\prime} \geq i, k \in K_{i^{\prime}} ; \\
& \text { for } k=1, W_{i^{\prime}}^{(k-1)}=W_{0 i} \\
& \sum_{i \in I} x_{i, j}{ }^{(k)} \leq u_{k} \quad \forall i^{\prime} \in I^{n e w}, k \in K_{i^{\prime}}, j \in J_{i, i^{\prime}}
\end{aligned}
$$

- Input/output volume balance - Due to liquid incompressibility, an exact balance between input and output volumes at every operation $k$ must be defined (Constraints 14).

$$
\sum_{\substack{i \in I_{j} \\ i \leq i^{\prime}}} \sum_{j \in J_{i, i^{\prime}}} D_{i, j}{ }^{(k)}=Q_{k} \quad \forall i^{\prime} \in I^{\text {new }}, k \in K_{i^{\prime}}
$$

- Fulfillment of the injection/delivery plan established at the aggregate level - The total volume injected into the pipeline and the total amount diverted from every batch $i^{\prime} \geq i$ must accomplish the aggregate plan (Constraints 15-16).

$$
\begin{array}{cc}
\sum_{k \in K_{i^{\prime}}} D_{i, j}{ }^{(k)}=d d_{i, j}{ }^{\left(i^{\prime}\right)} & \forall i \in I, i^{\prime} \in I^{\text {new }}, i^{\prime} \geq i, j \in J_{i, i^{\prime}} \\
\sum_{k \in K_{i^{\prime}}} Q_{k}=q q_{i^{\prime}} & \forall i^{\prime} \in I^{\text {new }}
\end{array}
$$

- Active and idle pipeline segments definition - Active and stopped pipeline volumes are identified to measure the solution performance (Constraints 17-19).

$$
\begin{gathered}
v_{j}{ }^{(k)} \geq \sum_{i \in I} x_{i, j}{ }^{(k)} \quad \forall j \in J_{i, i^{\prime}}, i^{\prime} \in I^{\text {new }}, k \in K_{i^{\prime}} \\
v_{j}{ }^{(k)} \leq \sum_{i \in I} \sum_{\substack{j^{\prime} \geq j \\
j^{\prime} \in J}} x_{i, j^{\prime}}{ }^{\left({ }^{\prime}\right)} \\
v_{j-1}{ }^{(k)} \geq v_{j}{ }^{(k)} \\
\forall j \in J_{i, i^{\prime}}, i^{\prime} \in I^{\text {new }}, k \in K_{i^{\prime}} \\
\forall j>1, k \in K
\end{gathered}
$$

- Identifying the farthest active segment - The location of the farthest terminal receiving product from the line is identified to obtain the stoppage and activation costs (Constraints 20-24).

$$
F A T_{k} \geq \sigma_{j} v_{j}{ }^{(k)} \quad \forall j \in J, k \in K
$$




$$
\begin{gathered}
F A T_{k} \leq \sigma_{j}+\left(p v-\sigma_{j}\right)\left(v_{j+1}{ }^{(k)}\right) \quad \forall j \in J, k \in K \\
A V_{k} \geq F A T_{k}-F A T_{k-1} \quad \forall k \in K_{i^{\prime}}, k>1, i^{\prime} \in I^{\text {new }} \\
S V_{k} \geq F A T_{k-1}-F A T_{k}+\sigma_{T A} \quad \forall i^{\prime} \in I^{\text {new }}, k \in K_{i^{\prime}}, k>1, i^{\prime} \in I^{\text {new }} \\
S V_{k} \geq F A T_{k}+\sigma_{T A} \quad \forall i^{\prime} \in I^{\text {new }}, k \in K_{i^{\prime}}, k=1, i^{\prime} \in I^{\text {new }}
\end{gathered}
$$

- Measuring pumping costs due to friction loss - The volume in motion at each pipeline segment $j$ is exactly equal to the overall quantity of product delivered to downstream terminals $j^{\prime} \geq j$ (Constraints 25).

$$
Q j_{j, k}=\sum_{i \in I} \sum_{\substack{j^{\prime} \in J_{i, i^{\prime}} \\ j^{\prime} \geq \geq j^{\prime}}} D^{(k)} \quad \forall i^{\prime} \in I^{\text {new }}, k \in K_{i^{\prime}}, j \in J
$$

If segment $j$ is in motion during operation $k$, the corresponding flow rate should be set to one of the ranges $r$ into which the flow rate admissible interval is divided (Constraints 26).

$$
\sum_{r \in R_{j}} y_{j, r}{ }^{(k)}=v_{j}{ }^{(k)} \quad \forall k \in K, j \in J
$$

The volume in motion along segment $j$ while performing the detailed operation $k$ exactly matches one of the variables $Q Q j_{j, r}{ }^{(k)}$ of the corresponding flow rate range $r$. Only one of the variables $Q Q j_{j, r}{ }^{(k)}$ will be positive for every $r$, since the flow rate must belong to only one of the admissible ranges $r$ (Constraints 27).

$$
\begin{aligned}
Q j_{j, k}=\sum_{r \in R_{j}} Q Q j_{j, r}{ }^{(k)} \forall k \in K, j \in J ; \quad Q Q j_{j, r}{ }^{(k)} \leq & Q_{\max } y_{j, r}{ }^{(k)} \\
& \forall k \in K, j \in J, r \in R_{j}
\end{aligned}
$$

The flow rate bounds at every range defined for a pipeline segment depend on the pipeline dimensions, and are given by the following constraints 28 .

$$
v r_{r, j}^{\min } L r_{j, r}{ }^{(k)} \leq Q Q j_{j, r}{ }^{(k)} \leq v r_{r, j}^{\max } L r_{j, r}{ }^{(k)} \quad \forall i^{\prime} \in I^{\text {new }}, k \in K_{i^{\prime}}, r \in R_{j}
$$

Variable $L r_{j, r}{ }^{(k)}$ equals the run duration $L_{k}$ if segment $j$ is active during operation $k$ and the volume pumped through $j$ moves at a flow rate belonging to range $r$. Otherwise, it takes a null value (Constraints 29-30).

Iberoamerican Journal of Industrial Engineering, Florianópolis, SC, Brasil, v. 5, n. 10, p. 115128, 2013. 


$$
\begin{aligned}
L r_{j, r}{ }^{(k)}-l^{\max }\left(1-y_{j, r}{ }^{(k)}\right) \leq L_{k} \leq L r_{j, r}{ }^{(k)}+l^{\max }\left(1-y_{j, r}{ }^{(k)}\right) & \\
& \forall k \in K, j \in J, r \in R_{j} \\
l^{\min } y_{j, r}{ }^{(k)} \leq L r_{j, r}{ }^{(k)} \leq l^{\max } y_{j, r}{ }^{\left({ }^{(}\right)} \quad & \forall k \in K, j \in J, r \in R_{j}
\end{aligned}
$$

As mentioned, a piecewise linear approximation is used to estimate the pumping energy cost incurred for transporting products into the pipeline during every operation $k$ (Constraints $31)$.

$$
P C_{k}=\sum_{j \in J} \sum_{r \in R_{j}}\left(a_{r, j} L r_{j, r}{ }^{(k)}+b_{r, j} Q Q j_{j, r}{ }^{(k)}\right) \quad \forall k \in K
$$

where $a_{r, j}$ and $b_{r, j}$ are the geometric coordinates of the piecewise linear approximations (D), in the slope-intercept form.

\subsection{Objective Function}

The aim of the present formulation is to obtain the optimal detailed schedule, which permits to simultaneously minimize energy consumption and stoppage/restart costs, through the minimum number of operations (Constraints 32).

$$
\text { Min } z=\sum_{k \in K}\left(c p P C_{k}+c s S V_{k}+c a A V_{k}+f c o u_{k}\right)
$$

\section{RESULTS AND DISCUSSION}

\subsection{A real world case study}

The example solved in this section takes as input data the aggregate operational plan of a single source pipeline with multiple offtake stations, presented by Cafaro and Cerdá (2008). This case study was first introduced by Rejowski and Pinto (2003). The problem goal is to find the optimal detailed schedule that exactly fulfills the input aggregate plan, at minimum total cost. As stated by the objective function, the aim is to minimize energy consumption, stoppage and restart costs. To demonstrate the efficiency of the proposed approach, the given solution will be compared with that obtained by Cafaro et al. (2012), in which the pumping costs were not taken into account. Figure 2 presents the aggregate schedule to be decomposed into detailed operations through the proposed MILP model. The length of the planning horizon is $660 \mathrm{~h}$, and 49 aggregate deliveries must be optimally scheduled at the detailed level.

Iberoamerican Journal of Industrial Engineering, Florianópolis, SC, Brasil, v. 5, n. 10, p. 115128, 2013. 
Figure 2 - Aggregate pipeline schedule for Example 1

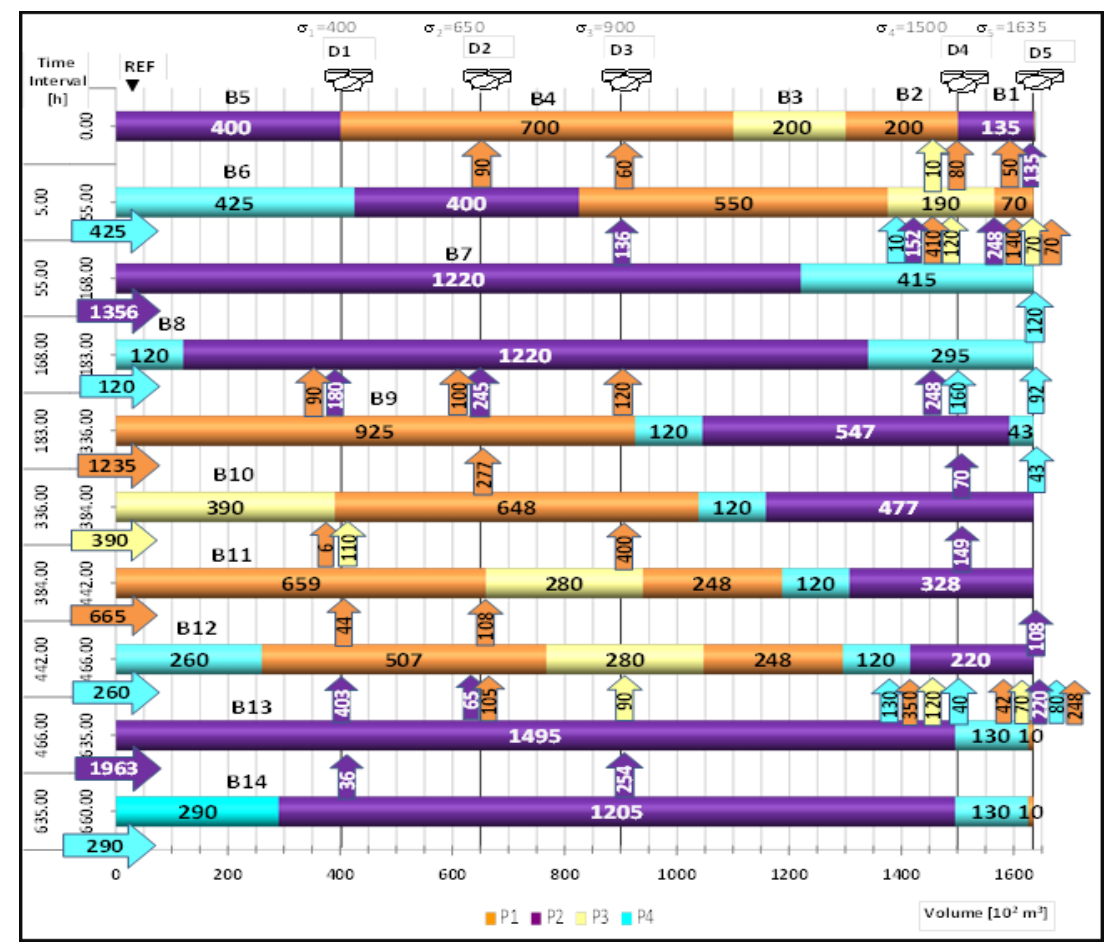

Source: Cafaro and Cerdá (2008)

Table 1 shows the admissible flow rate ranges for each segment. For making the piecewise linear approximations, the following eleven flow rate ranges are defined: r1: [100200]; r2: (200-300]; r3: (300-400]; r4: (400-500]; r5: (500-600]; r6: (600-700]; r7: (700-800]; r8: (800-900]; r9: (900-1000]; r10: (1000-1100]; r11: (1100-1200]. Lower bounds at ranges r2-r12 are slightly increased by a small positive value $\varepsilon$.

Table 1 - Admissible flow-rate ranges for each pipeline segment

\begin{tabular}{ccc}
\hline \multicolumn{1}{c}{ Pipeline Segment } & Flow Rate Range $\left(\mathbf{m}^{\mathbf{3}} / \mathbf{h}\right)$ & Possible Ranges \\
\hline Refinery - Terminal D1 & $700-1200$ & r7-r11 \\
Terminal D1 - Terminal D2 & $600-1200$ & r6-r11 \\
Terminal D2 - Terminal D3 & $600-1200$ & r6-r11 \\
Terminal D3 - Terminal D4 & $600-1200$ & r6-r11 \\
Terminal D4 - Terminal D5 & $400-800$ & r4-r7 \\
\hline
\end{tabular}

Figure 3 shows the optimal detailed schedule solution obtained by applying the MILP formulation presented in this work. Note that a total of 40 pumping runs are performed over the time horizon. 


\subsection{Comparing results}

The optimal detailed schedule derived from the proposed approach (Rate-Dependent Cost Model: RDC) is compared with the one found when only stoppage and restart costs are taken into account (Rate-Independent Cost Model: RIC). As shown in Figure 3, the aggregate plan is decomposed into 40 detailed operations. The major difference with regards to previous solutions is in the total number of deliveries. The new solution proposes 20 more partial deliveries ( 83 vs. 63 ), despite the total number of pumping runs are the same.

Figure 3 -Optimal detailed schedule introducing pumping costs

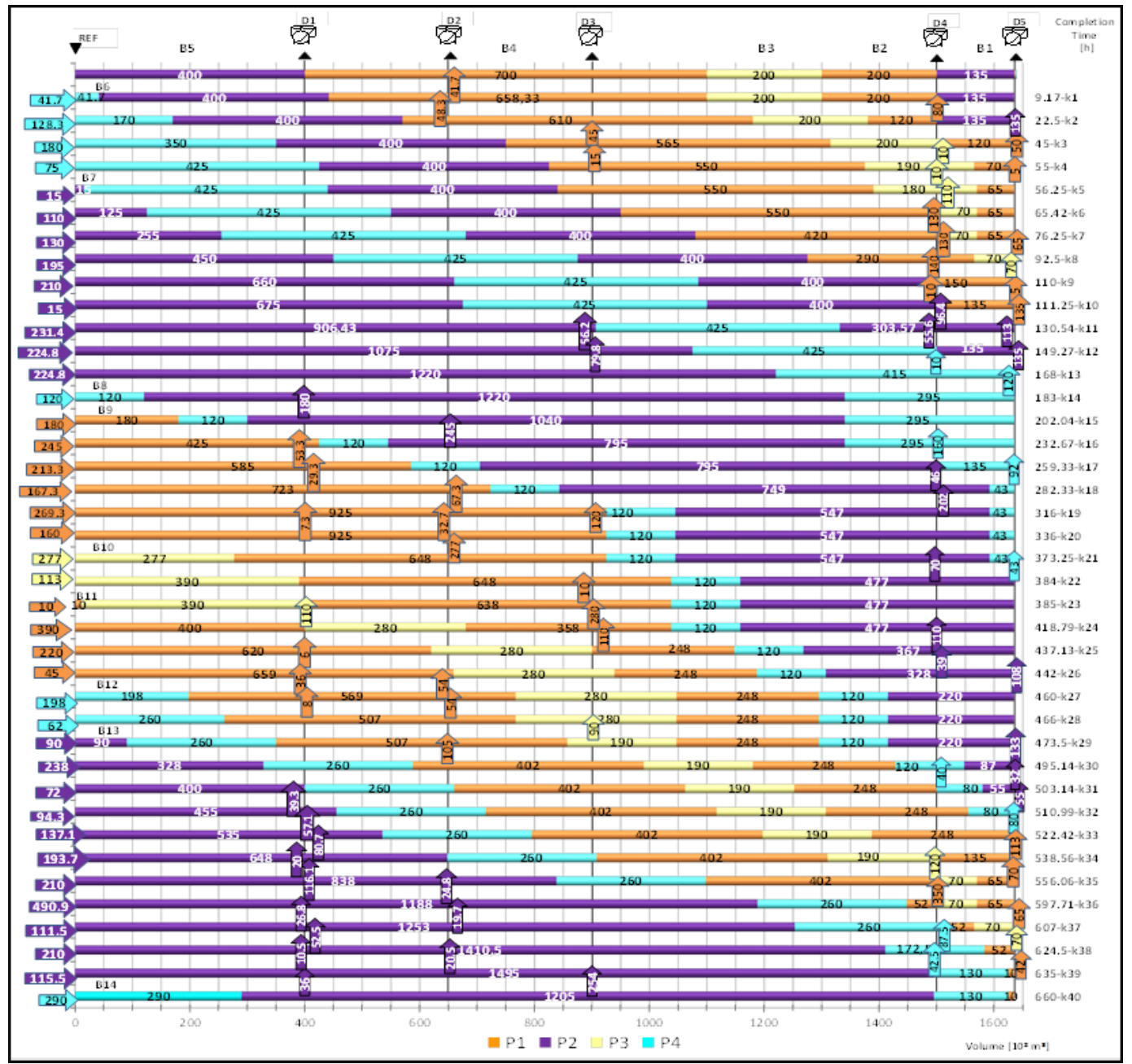

Source: Cafaro and Cerdá (2008)

When pumping energy costs are taken into account, the number of individual deliveries is higher, by making more simultaneous deliveries and thus reducing the flow rate at farthest segments. An illustration of this can be observed at Figure 3, when batch B9 is injected. The 90 units of product P1 demanded by depot D1 are supplied through three delivery operations,

Iberoamerican Journal of Industrial Engineering, Florianópolis, SC, Brasil, v. 5, n. 10, p. 115$128,2013$. 
while in the previous solution they are derived in only one operation. The same occurs with the 100 units of product P1 demanded by D2. Incorporating the pump-rate dependent energy costs, the best solution tends to develop smaller volume deliveries, and tries to maintain a stable and lower flow rates all along the pipeline in order to minimize the friction loss.

Figure 4 shows the variation of the flow rate with time in each pipeline segment, overlapping both solutions.

Figure 4 - Flow-rate variation in each pipeline segment: comparison of results

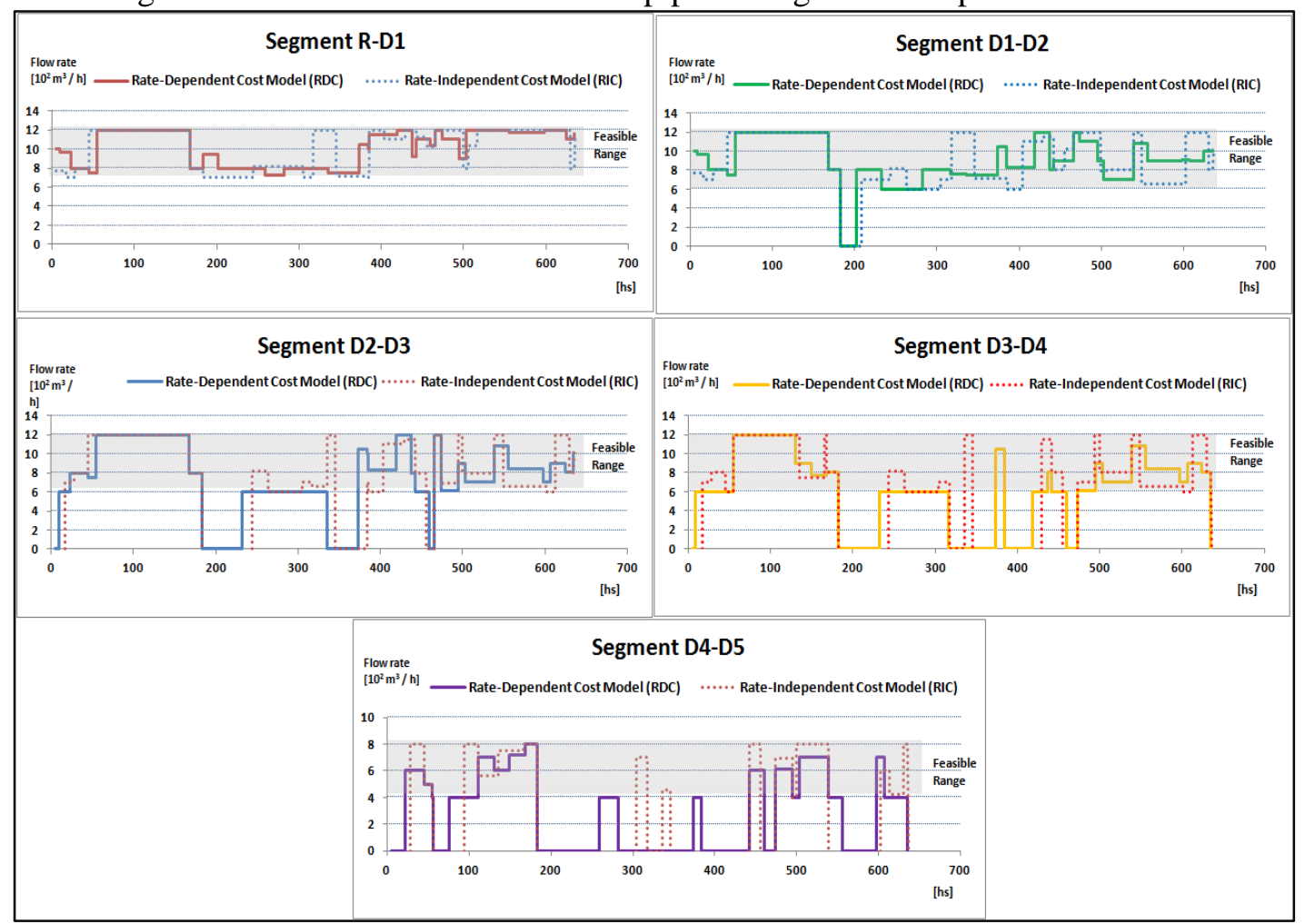

The most significant differences occur between time $\mathrm{t}=300 \mathrm{~h}$ and $\mathrm{t}=400 \mathrm{~h}$. At that interval, a noticeable separation of the flow-rate graphs arises. It is even more evident at segment D4-D5. Another important difference between both solutions is observed at segments D3-D4 and D4-D5 between time $\mathrm{t}=418.79 \mathrm{~h}$ and $\mathrm{t}=556.06 \mathrm{~h}$, when the detailed operations k24-k35 are executed. In general, the flow rate profile in the new solution is more stable all along the time-horizon. This directly affects the total operating costs, producing significant savings. Considering the energy costs, the savings amount to 1846.28 USD. Table 2 summarizes the results and the model performance, compared to previous approaches. 
Table 2 - Costs and Computational requirements

\begin{tabular}{ccccc}
\hline Cost Model & Pumping Cost [\$] & Restarting Cost [\$] & Total Cost [\$] & CPU time [s] \\
\hline RDC & 20545.09 & 81675.00 & 102220.09 & 2315.39 \\
RIC $^{[7]}$ & 22391.37 & 81675.00 & 104066.37 & 124.9 \\
\hline
\end{tabular}

\section{CONCLUSION}

An MILP model for the detailed scheduling of refined products pipelines introducing a novel piecewise linear approximation of the energy loss due to friction was developed. The optimal pump configuration is determined by considering a comprehensive objective function seeking for the minimization of the head loss along the pipeline, which is strongly dependent on the flow rate, and the reduction of segment stoppages and restarts.

Results obtained were compared to those reported in a previous work (CAFARO et al., 2012) in which the pumping costs were assumed to be independent from the pump rate. When pumping energy charges associated to the head loss inside the pipeline are taken into account, the flow rate is more stable all along the time-horizon, and important savings are obtained.

However, even with a rough division of the pump-rate range, the CPU time needed to achieve the optimal solution rises by a factor of 20. A further sensitivity analysis on the model performance and the solution quality variation with the pump rate range partitioning is proposed as future work.

\section{REFERENCES}

CAFARO, D.C.; CERDÁ, J. Dynamic Scheduling of Multiproduct Pipelines with Multiple Delivery Due Dates. Computers and Chemical Engineering, v. 32, p. 728-753, 2008.

CAFARO, V.G.; CAFARO, D.C.; MÉNDEZ C.A.; CERDÁ, J. Detailed Scheduling of Single-Source Pipelines with Simultaneous Deliveries to Multiple Offtake Stations. Industrial and Engineering Chemistry Research, v. 51, p. 6145-6165, 2012.

COLEBROOK, C.F.; WHITE, C. M. Experiments with fluid friction in roughened pipes. Proceedings..., of the Royal Society of London. Series A, Mathematical and Physical Sciences, v. 161, n. 906, p. 367-381, 1937.

LIANG, Y.T.; GONG J.; KANG, Z.L. Research on operation optimization of multi-product pipeline. Proceeding..., of the ASME International Pipeline Conference, 4-8 October 2004, Calgary, Alberta, Canada, p. 2055-2060, 2004.

MIESNER, T.O.; LEFFLER, W.L. Oil \& gas pipelines in nontechnical language. Pennwell: Tulsa, USA, 2006. 
REJOWSKI, R.; PINTO, J.M. A novel continuous time representation for the scheduling of pipeline systems with pumping yield rate constraints. Computers and Chemical Engineering, v. 32, p. 1042-1066, 2008.

REJOWSKI, R.; PINTO, J.M. Scheduling of a Multiproduct Pipeline System. Computers and Chemical Engineering, v. 27, p. 1229-1246, 2003.

Originais recebidos em: 26/10/2013

Aceito para publicação em: 15/04/2014 